\section{Traditional medicines: bridging the gaps for a better future}

\author{
Oliver Grundmann \\ Department of Medicinal Chemistry, \\ College of Pharmacy, University of \\ Florida, FL, USA
}

The use of traditional medicines has provided a crucial support and health system to millions of people around the globe for centuries past and this continues to the present day. In some countries, less than $20 \%$ of the population has access to basic generic medicines or healthcare. ${ }^{1}$ This highlights the need for a global initiative, such as the World Health Organization's Millenium Development Goals. This includes fighting child weight deficit, providing access to essential medicines, and treating diseases that significantly impact the health of the community, such as HIV/AIDS, cancer, malaria, and tuberculosis. ${ }^{1}$

Apart from these ambitious goals, there is a growing need for medicines and treatment approaches that are inexpensive, effective, and that are not associated with the high side effect burden that often limits conventional pharmacological therapy. With a slowing or even stagnant drug development pipeline in the pharmaceutical industry, many turn to traditional medicines in the search for better treatment options. Even in industrialized nations with significant healthcare access, over $40 \%$ of the population utilize complementary or alternative medicines (CAM) because of insufficient therapeutic outcomes with pharmacotherapy alone. 2,3 Furthermore, it has been evident throughout history that nature provides a wealth of compounds that have been utilized as medicines or at least as lead structures for the development of drugs.,45 Also, nutrition and a healthy lifestyle have a significant impact on our well-being.

The message is clear: nature can provide us with treatments for a wide variety of disorders. But for a multitude of reasons, there is conflicting opinion about how the messenger, the scientific community, is getting this message across. If CAM should be regarded as a valid therapeutic treatment approach, the current hurdles to overcome are being enforced by the respective regulatory agencies such as the EMA and the FDA.3,6

The strongest resistance against the use of CAM comes from the limited scientific data available to show the effectiveness of such treatments. This can be remedied by using preclinical and clinical study protocols that are rigid, reproducible, and provide sufficient statistical power to support the observed health effects. The second important milestone is safety. This was identified, for example, when Kava root (Piper methysticum, Piperaceae) was associated with hepatotoxicity ${ }^{7}$ and St. John's Wort (Hypericum perforatum, Clusiaceae) has been recognized as a contributing factor in the development of serotonin syndrome. ${ }^{8}$ Safety concerns affect CAM and conventional medical approaches alike. But CAM therapies often come under more scrutiny if side effects are reported because of the misguided belief that CAM treatments should be free of these phenomena. Every intervention can be associated with adverse effects and educating the public about this is an important component in ensuring safe and responsible use of CAM. The third, and probably most challenging, component in the scientific recognition of CAM is quality. Quality assurance and control measures are a crucial step towards ensuring consistency in effectiveness and safety. However, standardization of herbal medicines is often complicated by the limited knowledge about the compounds that contribute to the activity of the extract. Consistency in extract preparation and standardization to marker compounds is currently, therefore, the best approach and has already been accepted by the EMA and the FDA. ${ }^{9-11}$

Another important message in favor of CAM that is voiced more frequently these days is the fact that many chronic disorders (e.g. diabetes, cardiovascular diseases, hyperlipidemia, cancer) often require a polypharmacy approach combining a variety of medications that work synergistically but through different mechanisms. This is not new. Traditional medicines have long been known to contain a wide range of active principles with a variety of activities and many physicians would agree that a more personal relationship between healthcare provider and patient, a more holistic approach to caring for the patient and not limiting therapy to the disorder, often yields much better patient compliance and therapeutic outcomes.

After all, maybe the differences between complementary and alternative medical approaches and conventional Western medicine are not so great. There are certainly many ways in which we can help to bridge these differences and the scientific community can make a significant contribution towards reaching this goal.

\section{References}

1. Hogerzeil HV, Mirza Z. The World Medicines Situation 2011 - Access to Essential Medicines as Part of the Right to Health. Geneva: 2011.
Correspondence: Oliver Grundmann, Department of Medicinal Chemistry, College of Pharmacy, University of Florida, FL, USA

Received for publication: 15 September 2011. Accepted for publication: 15 September 2011.

This work is licensed under a Creative Commons Attribution NonCommercial 3.0 License (CC BYNC 3.0).

(C) Copyright O. Grundmann, 2011

Licensee PAGEPress, Italy

Alternative Medicine Studies 2011; 1:e13

doi:10.4081/ams.2011.e13

2. Barnes PM, Bloom B, Nahin RL. Complementary and alternative medicine use among adults and children: United States, 2007. Natl Health Stat Report 2008;12:1-23.

3. Robinson MM, Zhang X. The World Medicines Situtiation 2011 - Traditional Medicines: Global Situation, Issues and Challenges. Geneva: 2011.

4. Butler MS. Natural products to drugs: natural product-derived compounds in clinical trials. Nat Prod Rep 2008;25:475-516.

5. Carter GT. Natural products and Pharma 2011: Strategic changes spur new opportunities. Nat Prod Rep. 2011 Sep 12. [Epub ahead of print]

6. Anderson L, Briggs D, Cardini F, et al. General Guidelines for Methodologies on Research and Evaluation of Traditional Medicines. Geneva: World Health Organization; 2000. http://whqlibdoc.who. int/hq/2000/WHO_EDM_TRM_2000.1.pdf. Accessed: 09/15/2011.

7. Teschke R, Schulze J. Risk of kava hepatotoxicity and the FDA consumer advisory. JAMA 2010;304:2174-5.

8. Borrelli F, Izzo AA. Herb-drug interactions with St John's wort (Hypericum perforatum): an update on clinical observations. AAPS J 2009;11:710-27.

9. Barnes J. Quality, efficacy and safety of complementary medicines: fashions, facts and the future. Part I. Regulation and quality. Br J Clin Pharmacol 2003;55:226-33.

10. Rousseaux CG, Schachter H. Regulatory issues concerning the safety, efficacy and quality of herbal remedies. Birth Defects Res B Dev Reprod Toxicol 2003;68:505-10.

11. Cañigueral S, Tschopp R, Ambrosetti L, et al. The Development of Herbal Medicinal Products: Quality, Safety and Efficacy as Key Factors. Pharmaceutical Medicine 2008;22:107-18. 